\title{
Particle plasmons of metal nanospheres: Application of multiple scattering approach
}

\author{
Ruey-Lin Chern, ${ }^{1, *}$ Xing-Xiang Liu, ${ }^{1,2}$ and Chien-Cheng Chang ${ }^{2,1}$ \\ ${ }^{1}$ Institute of Applied Mechanics, National Taiwan University, Taipei 106, Taiwan, Republic of China \\ ${ }^{2}$ Division of Mechanics, Research Center for Applied Sciences, Academia Sinica, Taipei 115, Taiwan, Republic of China
}

(Received 24 November 2006; revised manuscript received 11 April 2007; published 30 July 2007)

\begin{abstract}
In this paper, we study particle plasmons associated with a chain of metal nanospheres by the method of multiple scattering. The extinction efficiency is used to identify the resonant modes in nanoparticle chains. Special emphasis is placed upon the multipolar nature of particle plasmons at two major resonant modes by studying the associated field patterns, surface charges, and distributions of the field enhancement. Effects of the number of particles, interparticle spacing, and particle alignment are investigated by examining the frequency shift, bandwidth, and the number of resonant modes.
\end{abstract}

\section{DOI: 10.1103/PhysRevE.76.016609}

\section{INTRODUCTION}

The optical properties of metal spheres have been the subject of continuous attention [1-3]. One of the most important features is the appearance of particle plasmons, which are resonant modes of the metal sphere coupled with the incident light. Particle plasmons differ from surface plasmon polaritons on planar surfaces [4] in two aspects. First, they are localized surface plasmons associated with metal particles that produce significant field enhancement on or near the particle surfaces. Their resonant behaviors depend not only on the intrinsic properties of the metal such as the bulk plasma frequency $\omega_{\mathrm{p}}$, but also on the particle geometry. Second, it is not necessary to use coupling techniques [5] such as prism, grating, aperture, or lattice coupling to provide extra momentum to excite surface plasmons on particles. In fact, the field patterns associated with particle plasmons can be decomposed as multipolar oscillations and eddy currents [6], both of which are fundamental modes attributed to spherical particles. For metal particles ranging in size from micrometers down to tens or hundreds of nanometers, particle plasmons would occur in the infrared, visible, or even ultraviolet regime. In these frequency ranges, particle plasmons or localized surface plasmons have found various applications in miniaturized photonic and plasmonic devices [7,8], surface-enhanced Raman scattering [9-11], biosensors [12], nanolithography $[13,14]$, and optical data storage $[15,16]$.

Particle plasmons for single nanoparticles have been studied intensively based on the Mie theory [2]. Theoretically, there are an infinite number of resonant modes [17]. As the particle size increases, particle plasmon resonances move to longer wavelengths and become broadened, and additional higher-order modes are excited [2]. For nonregular shapes of particles, even more distinct resonances along with dramatic field enhancements were observed [18,19]. For nanoparticle pairs or arrays, interparticle coupling plays a major role in the properties of particle plasmons. Surface modes for two particles have been calculated using bispherical coordinates [20], and their optical properties were compared with different theories [21]. The coupling effects of particle pairs were

\footnotetext{
*chern@iam.ntu.edu.tw
}

PACS number(s): 42.25.Bs, 73.20.Mf, 73.63.-b, 78.67.-n

also studied by the scattering of fast electron beams $[22,23]$ and discussed based on the normal-mode theory [24,25]. In addition, collective excitation in an infinite set of aligned nanoparticles has been reported [26].

One of the important features of coupling between nanoparticles is the spectral shift as a function of separation distance. Experiments in light scattering spectra and numerical calculations by the finite-difference time-domain method also indicated the spectral shifts of the resonances [27]. In addition, the electromagnetic fields are strongly enhanced near the gap $[28,29]$, with optical forces induced between nanoparticles [30]. The optical properties of particle plasmons have also been investigated in the interparticle coupling [31-36] and the influence of size and shape [37]. Other interesting issues include the electromagnetic energy transport in nanoparticle chains [38-41], optical pulse propagation in nanoparticle waveguides [42,43], and surface plasmon dispersion relations [44]. More recently, the hybridization method has been proposed to describe the interaction of plasmon modes and calculate the plasmon energies of plasmonic nanostructures [45-48].

Particle plasmons for single nanoparticles and particle pairs were studied fairly completely. In contrast, relatively few efforts have been devoted to more particles except the references [38-44] cited above. In the present study, we investigate particle plasmons of metal nanospheres with major emphasis on local field enhancement and the multipolar nature of particle plasmons at major resonant modes. Particle plasmons associated with a chain of metal nanospheres are studied using the multiple scattering method [49,50]. This method is a semianalytical approach built upon the multiple expansion theory (or Mie theory) and the addition theorem for electromagnetic fields. In this approach, the multiple spheres problem is treated in effect as a single sphere subjected to an equivalent incident field that combines all scattered fields from other spheres with the original incident field. This approach involves a transformation between the incident and scattered fields, and the addition theorem serves this purpose. Vector spherical harmonics are employed as the bases for expanding the electromagnetic fields about the sphere centers. Boundary conditions on the nanospheres are exactly satisfied by the spherical harmonics. This mathematical skill is particularly useful in studying particle plasmons of spherical nanoparticles. In physical terms, the multipolar nature of particle plasmons is closely related to spherical 


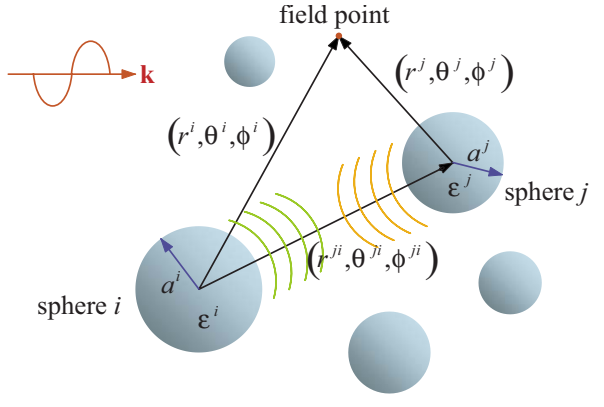

FIG. 1. (Color online) Schematic diagram of the multiple scattering approach for multiple nanospheres.

harmonics, in which the expansion orders directly correspond to dipoles, quadrupoles, octopoles, and so forth. The dielectric constants of metal nanoparticles are taken from experiments, in which the effect of electronic interband transitions was included. For smaller nanoparticles, the surface effect on the nanoscales is also considered with correction based on the metal's mean free path [51,52]. Particle plasmons are identified by locating the peaks of the extinction efficiency. The associated field patterns, surface charges, and distributions of the field enhancement are used to explore the multipolar nature of plasmon resonances in nanoparticle chains. Effects of the number of particles, interparticle spacing, and particle alignment are studied by examining the frequency shift, bandwidth, and the number of resonant modes in the extinction spectra.

\section{MULTIPLE SCATTERING APPROACH}

The multiple scattering method for electromagnetic waves is built upon the multiple expansion theory (or Mie theory), with a delicate use of the addition theorem for spherical harmonics. This approach has been developed by Mackowski [49] and Fuller [50], who employed the addition theorem in a somewhat different way. Here, we organize and report the formalism of the multiple scattering approach in a more compact manner, and added explanatory notes in physical terms wherever necessary to elucidate the theorem and equations used in this approach.

The problem under study here is the scattering of an incident plane wave on $N_{s}$ arbitrarily located metal spheres. Figure 1 shows the schematic diagram of this problem. For the sphere $i$ of radius $a^{i}$ with dielectric constant $\varepsilon^{i}(i$ $=1, \ldots, N_{s}$ ), the time-harmonic (with time dependence $e^{-i \omega t}$ ) incident electric field $\mathbf{E}_{0}$, scattered electric field $\mathbf{E}_{s}^{i}$, and internal electric field $\mathbf{E}_{1}^{i}$ can be expanded as $[49,50]$

$$
\begin{aligned}
& \mathbf{E}_{0}=\sum_{n=1}^{\infty} \sum_{m=-n}^{n}\left[p_{m n}^{i} \mathbf{N}_{m n}^{(1)}+q_{m n}^{i} \mathbf{M}_{m n}^{(1)}\right], \\
& \mathbf{E}_{s}^{i}=\sum_{n=1}^{\infty} \sum_{m=-n}^{n}\left[a_{m n}^{i} \mathbf{N}_{m n}^{(3)}+b_{m n}^{i} \mathbf{M}_{m n}^{(3)}\right], \\
& \mathbf{E}_{1}^{i}=\sum_{n=1}^{\infty} \sum_{m=-n}^{n}\left[d_{m n}^{i} \mathbf{N}_{m n}^{(1)}+c_{m n}^{i} \mathbf{M}_{m n}^{(1)}\right],
\end{aligned}
$$

where

$$
\begin{gathered}
\mathbf{M}_{m n}^{(\alpha)}=\boldsymbol{\nabla} \times \mathbf{r} u_{m n}^{(\alpha)}, \\
\mathbf{N}_{m n}{ }^{(\alpha)}=\frac{1}{k} \boldsymbol{\nabla} \times \mathbf{M}_{m n}^{(\alpha)},(\alpha=1,3)
\end{gathered}
$$

are two mutually orthogonal vector spherical harmonics, generated from the scalar functions $u_{m n}^{(1)}=j_{n}(r) P_{n}^{m}(\cos \theta) e^{i m \phi}$ and $u_{m n}^{(3)}=h_{n}^{(1)}(r) P_{n}^{m}(\cos \theta) e^{i m \phi}$. These two functions are products of the decoupled components in $r, \theta$, and $\phi$ coordinates, in which $j_{n}(r), h_{n}^{(1)}(r)$, and $P_{n}^{m}(\cos \theta)$ are the spherical Bessel function, the spherical Hankel function of the first kind, and the associated Legendre function, respectively. In Eqs. (1)-(3), $\quad \mathbf{E}_{0}=\mathbf{E}_{0}\left(r^{i}, \theta^{i}, \phi^{i}\right), \quad \mathbf{E}_{s}^{i}=\mathbf{E}_{s}^{i}\left(r^{i}, \theta^{i}, \phi^{i}\right), \quad$ and $\quad \mathbf{E}_{1}^{i}$ $=\mathbf{E}_{1}^{i}\left(n^{i} r^{i}, \theta^{i}, \phi^{i}\right)$, where $n^{i}=\sqrt{\varepsilon^{i}}$ is the refractive index of the sphere $i$. Note that the arguments are the relative spherical coordinates of the field point about the center of the sphere $i$. The expansion coefficients $p_{m n}^{i}$ and $q_{m n}^{i}$ in Eq. (1) will be prescribed by the incident fields, while $a_{m n}^{i}$ and $b_{m n}^{i}$ in Eq. (2), and $d_{m n}^{i}$ and $c_{m n}^{i}$ in Eq. (3) are to be determined by boundary conditions. Similar expansions for the corresponding incident magnetic field $\mathbf{H}_{0}$, scattered magnetic field $\mathbf{H}_{s}^{i}$, and internal magnetic field $\mathbf{H}_{1}^{i}$ about the center of the sphere $i$ can be obtained by using Faraday's law: $\boldsymbol{\nabla} \times \mathbf{E}=i \omega \mathbf{H}$.

In this formalism, the transversality conditions for electromagnetic fields (in source-free problems) $\boldsymbol{\nabla} \cdot \mathbf{E}=0$ and $\boldsymbol{\nabla} \cdot \mathbf{H}=0$ are automatically satisfied in virtue of $\boldsymbol{\nabla} \cdot \mathbf{M}_{m n}^{(\alpha)}=0$ and $\boldsymbol{\nabla} \cdot \mathbf{N}_{m n}^{(\alpha)}=0$. It follows that two mutually orthogonal bases $\mathbf{M}_{m n}^{(\alpha)}$ and $\mathbf{N}_{m n}^{(\alpha)}$ suffice to describe the whole electromagnetic fields. Note that $\mathbf{M}_{m n}^{(\alpha)}$ is everywhere tangential to the surface of the sphere $\left(\mathbf{r} \cdot \mathbf{M}_{m n}^{(\alpha)}=0\right)$ and corresponds to the eddy current (magnetic mode) [6], while $\mathbf{N}_{m n}^{(\alpha)}$ is perpendicular to $\mathbf{M}_{m n}^{(\alpha)}$ and depicts the dipole, quadrupole, octopole, and so forth (electric mode) [2]. For a plane incident wave $\mathbf{E}_{0}$ $=E_{0} e^{i k r^{i} \cos \theta^{i}} \hat{x}$, the two expansion coefficients in Eq. (1) are given as [53] $p_{1 n}^{i}=-\frac{1}{2} i^{n+1} \frac{2 n+1}{n(n+1)} e^{i k r^{i} \cos \theta^{i}}, \quad p_{-1 n}^{i}=\frac{1}{2} i^{n+1}(2 n$ $+1) e^{i k r^{i} \cos \theta^{i}}, \quad q_{1 n}^{i}=p_{1 n}^{i}, \quad q_{-1 n}^{i}=-p_{-1 n}^{i}$, and $p_{m n}^{i}=q_{m n}^{i}=0$ for $|m| \neq 1$. The four coefficients in Eqs. (2) and (3) are determined by continuity of the tangential electric and magnetic field components (in $\theta$ and $\phi$ directions) on the surface of the sphere $i$ :

$$
\begin{aligned}
\left(\mathbf{E}_{0}\right)_{\theta, \phi}+\left(\mathbf{E}_{s}^{\mathrm{all}}\right)_{\theta, \phi} & =\left(\mathbf{E}_{1}^{i}\right)_{\theta, \phi}, \\
\left(\mathbf{H}_{0}\right)_{\theta, \phi}+\left(\mathbf{H}_{s}^{\mathrm{all}}\right)_{\theta, \phi} & =\left(\mathbf{H}_{1}^{i}\right)_{\theta, \phi} .
\end{aligned}
$$

It is noticed that the total scattered fields $\mathbf{E}_{s}^{\text {all }}$ and $\mathbf{H}_{s}^{\text {all }}$ are obtained by adding together all the scattered fields from each sphere: $\mathbf{E}_{s}^{\text {all }}=\sum_{j=1}^{N_{s}} \mathbf{E}_{s}^{j}$ and $\mathbf{H}_{s}^{\text {all }}=\sum_{j=1}^{N_{s}} \mathbf{H}_{s}^{j}$. Therefore the boundary conditions for the sphere $i$ may involve a combination of $\mathbf{M}_{m n}^{(3)}$ and $\mathbf{N}_{m n}^{(3)}$ about the centers of the spheres $j=1, \ldots, N_{s}$ $(j \neq i)$. As a result, simple equations for the expansion coefficients are not attainable at this stage. One way to resolve this difficulty is to use the addition theorem [49]:

$$
\mathbf{M}_{m n}^{(3)}=\sum_{l=1}^{\infty} \sum_{k=-l}^{l}\left[A_{k l}^{m n} \mathbf{M}_{k l}^{(1)}+B_{k l}^{m n} \mathbf{N}_{k l}^{(1)}\right]
$$




$$
\mathbf{N}_{m n}^{(3)}=\sum_{l=1}^{\infty} \sum_{k=-l}^{l}\left[A_{k l}^{m n} \mathbf{N}_{k l}^{(1)}+B_{k l}^{m n} \mathbf{M}_{k l}^{(1)}\right],
$$

where $\mathbf{M}_{m n}^{(3)}\left(r^{j}, \theta^{j}, \phi^{j}\right)$ and $\mathbf{N}_{m n}^{(3)}\left(r^{j}, \theta^{j}, \phi^{j}\right)$ are functions about the center of the sphere $j$, and $\mathbf{M}_{k l}^{(1)}\left(r^{i}, \theta^{i}, \phi^{i}\right)$ and $\mathbf{N}_{k l}^{(1)}\left(r^{i}, \theta^{i}, \phi^{i}\right)$ are functions about the center of the sphere $i$. The coefficients $A_{k l}^{m n}\left(r^{j i}, \theta^{j i}, \phi^{j i}\right)$ and $B_{k l}^{m n}\left(r^{j i}, \theta^{j i}, \phi^{j i}\right)$ depend on the relative coordinates of the center of the sphere $j$ with respect to the center of the sphere $i$ (cf. Fig. 1), and can be explicitly determined as follows [49]:

$$
\begin{aligned}
A_{k l}^{m n}= & \frac{1}{2 l(l+1)}\left[(l-k)(l+k+1) C_{k+1 l}^{m+1 n}+2 m k C_{k l}^{m n}\right. \\
& \left.+(n+m)(n-m+1) C_{k-1 l}^{m-1 n}\right], \\
B_{k l}^{m n}= & -\frac{i(2 l+1)}{2 l(l+1)(2 l-1)}\left[(l-k)(l-k-1) C_{k+1 l-1}^{m+1 n}\right. \\
& \left.+2 m(l-k) C_{k l-1}^{m n}-(n+m)(n-m+1) C_{k-1 l-1}^{m-1 n}\right],
\end{aligned}
$$

where $C_{k l}^{m n}$ are related to each other by several recurrence relations with the starting coefficient

$$
\begin{gathered}
C_{k l}^{00}=(-1)^{k+l}(2 l+1) h_{l}^{(1)}\left(r^{j i}\right) P_{l}^{-k}\left(\cos \theta^{i i}\right) e^{-i k \phi^{j i}}, \\
C_{k l}^{m n}=0 \text { if }|m|>n \text { or }|k|>l .
\end{gathered}
$$

In Eqs. (8) and (9), each outgoing spherical harmonic $\mathbf{M}_{m n}^{(3)}$ or $\mathbf{N}_{m n}^{(3)}$ about the sphere $j$ is replaced by a sum of standing spherical harmonics $\mathbf{M}_{k l}^{(1)}$ and $\mathbf{N}_{k l}^{(1)}$ about the sphere $i$. In mathematical terms, the expansion center of the spherical harmonic is translated from the sphere $j$ to the sphere $i$. In physical terms, a scattered wave from the sphere $j$ is converted to a sum of incident waves to the sphere $i$. The transformation between the sphere $j$ and sphere $i$ is dependent on the geometric relations specified by the relative coordinates $\left(r^{j i}, \theta^{i j}, \phi^{j i}\right)$.

Using the addition theorem (8) and (9) for the scattered waves in Eq. (2) and applying the boundary conditions (6) and (7) on the surface of the sphere $i$ yield [49]

$$
\begin{aligned}
& a_{m n}^{i}=-\alpha_{n}^{i}\left[p_{m n}^{i}+\sum_{\substack{j=1 \\
j \neq i}}^{N_{s}} \sum_{l=1}^{\infty} \sum_{k=-l}^{l}\left(A_{m n}^{k l} a_{k l}^{j}+B_{m n}^{k l} b_{k l}^{j}\right)\right], \\
& b_{m n}^{i}=-\beta_{n}^{i}\left[q_{m n}^{i}+\sum_{\substack{j=1 \\
j \neq i}}^{N_{s}} \sum_{l=1}^{\infty} \sum_{k=-l}^{l}\left(A_{m n}^{k l} b_{k l}^{j}+B_{m n}^{k l} a_{k l}^{j}\right)\right],
\end{aligned}
$$

where

$$
\begin{aligned}
& \alpha_{n}^{i}=\frac{n^{i} \psi_{n}\left(n^{i} x^{i}\right) \psi_{n}^{\prime}\left(x^{i}\right)-\psi_{n}\left(x^{i}\right) \psi_{n}^{\prime}\left(n^{i} x^{i}\right)}{n^{i} \psi_{n}\left(n^{i} x^{i}\right) \xi_{n}^{\prime}\left(x^{i}\right)-\xi_{n}\left(x^{i}\right) \psi_{n}^{\prime}\left(n^{i} x^{i}\right)}, \\
& \beta_{n}^{i}=\frac{\psi_{n}\left(n^{i} x^{i}\right) \psi_{n}^{\prime}\left(x^{i}\right)-n^{i} \psi_{n}\left(x^{i}\right) \psi_{n}^{\prime}\left(n^{i} x^{i}\right)}{\psi_{n}\left(n^{i} x^{i}\right) \xi_{n}^{\prime}\left(x^{i}\right)-n^{i} \xi_{n}\left(x^{i}\right) \psi_{n}^{\prime}\left(n^{i} x^{i}\right)} .
\end{aligned}
$$

Here, $\psi_{n}\left(x^{i}\right)=x^{i} j_{n}\left(x^{i}\right)$ and $\xi_{n}\left(x^{i}\right)=x^{i} h_{n}^{(1)}\left(x^{i}\right)$ are the RiccatiBessel functions with $x^{i}=k a^{i}$, and the prime denotes deriva- tive with respect to the argument. Let $N_{\max }$ be the maximum order for expansion of the spherical harmonics, the coefficients $a_{m n}^{i}$ and $b_{m n}^{i}$ for all spheres and orders $\left(i=1, \ldots, N_{s}\right.$, $m=-n, \ldots, n$, and $\left.n=1, \ldots, N_{\max }\right)$ are put together to form a matrix system. In tensor form, we have $\mathbf{a}^{i}+\sum_{j=1, j \neq i}^{N_{s}} \mathbf{T}^{j i} \mathbf{a}^{j}=\mathbf{p}^{i}$, where $\mathbf{a}^{i}=\left(a_{m n}^{i}, b_{m n}^{i}\right), \mathbf{p}^{i}=-\left(\alpha_{n}^{i} p_{m n}^{i}, \beta_{n}^{i} q_{m n}^{i}\right)$, and $\mathbf{T}^{i}$ represents a transformation matrix from the sphere $j$ to the sphere $i$. After solving $a_{m n}^{i}$ and $b_{m n}^{i}$ for the scattered fields in Eq. (2), the other two expansion coefficients for the internal fields in Eq. (3) are given by $c_{m n}^{i}=\gamma_{n}^{i} q_{m n}^{i}$ and $d_{m n}^{i}=\delta_{n}^{i} p_{m n}^{i}$, where

$$
\begin{aligned}
& \gamma_{n}^{j}=\frac{i n^{i}}{n^{i} \psi_{n}\left(n^{i} x^{i}\right) \xi_{n}^{\prime}\left(x^{i}\right)-\xi_{n}\left(x^{i}\right) \psi_{n}^{\prime}\left(n^{i} x^{i}\right)}, \\
& \delta_{n}^{i}=\frac{i n^{i}}{\psi_{n}\left(n^{i} x^{i}\right) \xi_{n}^{\prime}\left(x^{i}\right)-n^{i} \xi_{n}\left(x^{i}\right) \psi_{n}^{\prime}\left(n^{i} x^{i}\right)} .
\end{aligned}
$$

Note that for each sphere $i$ the whole electromagnetic fields are now represented solely in terms of the absolute coordinates about the sphere $i$. The underlying problem can therefore be treated in effect as a single sphere subjected to an equivalent incident field that combines all scattered fields from other spheres with the incident field. It is also noticed that the geometric relation between each pair of spheres has been considered in the coefficients $A_{k l}^{m n}$ and $B_{k l}^{m n}$, and the heart of this approach is the delicate use of the addition theorem [Eqs. (8) and (9)] for taking into account the scattered fields from multiple spheres.

Having obtained all the expansion coefficients: $p_{m n}^{i}, q_{m n}^{i}$, $a_{m n}^{i}, b_{m n}^{i}, c_{m n}^{i}$, and $d_{m n}^{i}$, the total electric (magnetic) field outside the spheres is obtained by adding together the incident field $\mathbf{E}_{0}\left(\mathbf{H}_{0}\right)$ and the scattered fields $\mathbf{E}_{s}^{j}\left(\mathbf{H}_{s}^{j}\right)$ from all spheres $j=1, \ldots, N_{s}$, and the field inside each individual sphere $i$ is simply $\mathbf{E}_{1}^{i}\left(\mathbf{H}_{1}^{i}\right)$. These coefficients are also used to calculate the extinction and absorption cross sections for each sphere $i$ through [49]

$$
\begin{aligned}
C_{\mathrm{ext}}^{i}= & \frac{2 \pi}{k^{2}} \operatorname{Re} \sum_{n=1}^{\infty} \sum_{m=-n}^{n} S_{m n}\left(a_{m n}^{i} p_{m n}^{*_{i}}+b_{m n}^{i} q_{m n}^{*_{i}}\right), \\
C_{\mathrm{abs}}^{i}= & \frac{2 \pi}{\left|n^{i}\right|^{2} k^{2}} \operatorname{Re} \sum_{n=1}^{\infty} \sum_{m=-n}^{n} S_{m n} \psi_{n}^{\prime}\left(n^{i} x^{i}\right) \psi_{n}^{*}\left(n^{i} x^{i}\right) \\
& \times\left(n^{i^{*}}\left|d_{m n}^{i}\right|^{2}+n^{i}\left|c_{m n}^{i}\right|^{2}\right),
\end{aligned}
$$

where $S_{m n}=\frac{n(n+1)}{2 n+1} \frac{(n+m) !}{(n-m) !}$, and the asterisk denotes the complex conjugate. The total extinction and absorption cross sections are then given by $C_{\mathrm{ext}}=\sum_{j=1}^{N_{s}} C_{\mathrm{ext}}^{j}$ and $C_{\mathrm{abs}}=\sum_{j=1}^{N_{s}} C_{\mathrm{abs}}^{j}$, respectively, and the total scattering cross section can be obtained either by $C_{\mathrm{sca}}=C_{\mathrm{ext}}-C_{\mathrm{abs}}$ or by the following equation [49]:

$$
C_{\mathrm{sca}}=\frac{2 \pi}{k^{2}} \sum_{n=1}^{\infty} \sum_{m=-n}^{n} S_{m n}\left(\left|a_{m n}^{\text {all }}\right|^{2}+\left|b_{m n}^{\text {all }}\right|^{2}\right),
$$

where

$$
a_{m n}^{\text {all }}=a_{m n}^{i}+\sum_{\substack{j=1 \\ j \neq i}}^{N_{s}} \sum_{l=1}^{\infty} \sum_{k=-l}^{l}\left(A_{m n}^{k l} a_{k l}^{i}+B_{k l}^{m n} b_{k l}^{i}\right),
$$




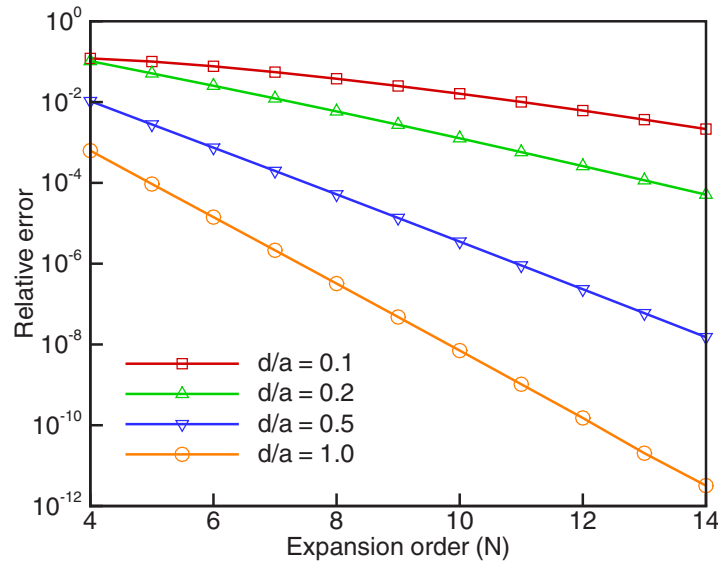

FIG. 2. (Color online) Convergence of the extinction efficiencies for two silver nanoparticles of radius $a=25 \mathrm{~nm}$ with various interparticle spacing $d / a$. The wavelength of the incident wave is $360 \mathrm{~nm}$.

$$
b_{m n}^{\mathrm{all}}=b_{m n}^{i}+\sum_{\substack{j=1 \\ j \neq i}}^{N_{s}} \sum_{l=1}^{\infty} \sum_{k=-l}^{l}\left(A_{m n}^{k l} b_{k l}^{i}+B_{k l}^{m n} a_{k l}^{i}\right) .
$$

In order to obtain the optical properties for spheres of different sizes and/or numbers, $C_{\text {ext }}, C_{\text {abs }}$, and $C_{\text {sca }}$ are normalized by the geometric cross section $A$ of the spheres to give the extinction, absorption, and scattering efficiencies: $Q_{\text {ext }}, Q_{\text {abs }}$, and $Q_{\text {sca }}$, respectively. For a single sphere or $N_{s}$ identical spheres aligned parallel to the incident electric field, $A$ $=N_{s} \pi a^{2}$, while for identical spheres aligned normal to the incident electric field, $A=\pi a^{2}$ in spite of the number of particles.

The multiple scattering approach provides an efficient way to investigate particle plasmons, in particular, for spherical nanoparticles. This is a semianalytical approach that maximizes the use of spherical harmonics with the advantage of boundary conditions being exactly satisfied on the particle surface. At infinity, these harmonics decay to zero. This property is particular, useful for particle plasmons where the electromagnetic fields are localized and enhanced near the particle surface. Roughly speaking, the order of expansions needed to achieve certain accuracy for single particles is linearly proportional to the electrical size $k a$ of the spheres. For multiple particles, higher-order expansions are necessary to obtain good accuracy for small interparticle spacings. Figure 2 shows the convergence of the extinction efficiencies for two silver nanoparticles of radius $a=25 \mathrm{~nm}$ with various interparticle spacing $d / a$. The wavelength of the incident wave is $360 \mathrm{~nm}$, and the relative error is defined as

$$
(\text { Error })=\frac{Q_{\mathrm{ext}}^{N}-Q_{\mathrm{ext}}^{N-1}}{Q_{\mathrm{ext}}^{N-1}},
$$

where the superscript $N$ denotes the order of expansions. It is shown that more expansion terms are needed for smaller interparticle spacings. For $N=10$, there are totally $\sum_{n=1}^{N}(2 n$ $+1)=N^{2}+2 N=120$ terms of expansions. In this case, the relative error (14) of the extinction efficiency for the most critical case of $d / a=0.1$ (where the interparticle spacing is only $2.5 \mathrm{~nm}$ ) is still below $5 \%$. For larger $d / a$, the relative errors decrease rapidly. In short, the additional theorem requires enough bases to convert the scattered field from one sphere into the incident field for another. The only restriction for the addition theorem to be valid is that each sphere cannot contact or even overlap with other spheres.

\section{RESULTS AND DISCUSSION}

In the present study, the experimentally obtained dielectric constant $\varepsilon_{\text {expt }}$ is used for silver nanoparticles [54]. The dielectric constant can be modeled as a sum of the contribution from the Drude dielectric function (due to the free electrons) and the contribution from the electronic interband transitions (due to the bound $d$-electrons) $[52,55]$ :

$$
\varepsilon_{\text {expt }}=1-\frac{\omega_{\mathrm{p}}^{2}}{\omega^{2}+i \gamma_{\mathrm{bulk}} \omega}+\varepsilon_{\mathrm{ib}},
$$

where $\omega_{\mathrm{p}}$ is the bulk plasma frequency and $\gamma_{\text {bulk }}$ is the bulk damping constant. For silver, $\omega_{\mathrm{p}}=1.4 \times 10^{16} 1 / \mathrm{s}$ and $\gamma_{\text {bulk }}$ $=3.2 \times 10^{13} 1 / \mathrm{s}[51]$.

For smaller nanoparticles with sizes down to the order of bulk mean free path $l_{\infty}$ of the electrons (for silver, $l_{\infty}$ $=52 \mathrm{~nm}$ [3]), additional damping due to collision of the electrons with the particle surface must be considered. To account for this phenomenon, a correction to $\gamma_{\text {bulk }}$ in the dielectric function has been proposed $[51,56]$ :

$$
\varepsilon=\varepsilon_{\text {expt }}+\frac{\omega_{\mathrm{p}}^{2}}{\omega^{2}+i \gamma_{\mathrm{bulk}} \omega}-\frac{\omega_{\mathrm{p}}^{2}}{\omega^{2}+i \gamma \omega},
$$

where

$$
\gamma=\gamma_{\mathrm{bulk}}+A v_{f} / a
$$

is the corrected damping constant, $A$ is a constant and usually taken as $1, a$ is the radius of the sphere, and $v_{f}$ is the Fermi velocity, which for silver $v_{f}=1.39 \times 10^{8} \mathrm{~cm} / \mathrm{s}$ [57]. Size correction is necessary for smaller particles at longer wavelengths. Nevertheless, for a particle radius near $50 \mathrm{~nm}$ (particle diameter $\approx 2 l_{\infty}$ ) or larger, the size effect becomes less important and the bulk dielectric constants are adequate for calculating the optical properties of nanoparticles. A detailed description of the surface effect for small metal spheres can be found in Ref. [52].

Plasmon resonances for nanoparticles correspond to peaks of the extinction spectrum. This is because the electrons in metal particles are now driving at their resonant frequency with relatively large oscillation amplitude, and correspondingly a large amount of energy is dissipated by the damping mechanism. Meanwhile, enhanced electromagnetic fields due to particle plasmon resonance with oscillating electric dipoles may radiate a substantial amount of energy to infinity in the form of scattering. To begin with, consider two nanoparticles aligned parallel to the incident electric field. Figure 3 shows the extinction efficiencies $Q_{\text {ext }}$ for two silver nanoparticles of radius $a=50 \mathrm{~nm}$ with various interparticle spacing from $d / a=0.1-5$. When the interparticle spacing is increased above $d / a=5$, the coupling between two nanopar- 


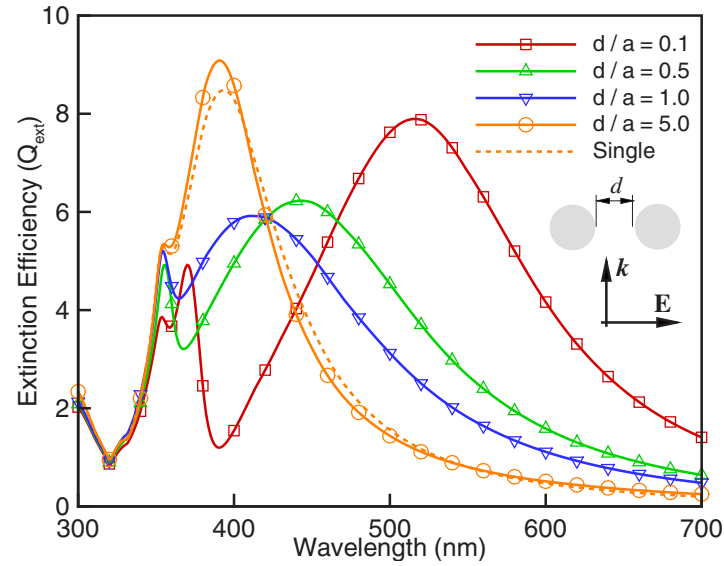

FIG. 3. (Color online) Extinction efficiencies for two silver nanoparticles of radius $a=50 \mathrm{~nm}$ with interparticle spacing $d / a$ $=0.1,0.5,1$, and 5 . The dashed line is the result of a single nanoparticle of the same size.

ticles becomes rather weak. However, the interference effect between the two nanoparticles is still present. Note that the extinction efficiency $Q_{\mathrm{ext}}$ for $d / a=5$ approaches that for the single nanoparticle of the same size (the dashed line in Fig. $3)$. On the other hand, at smaller interparticle spacings, more resonant peaks in the extinction spectrum appear. Figure 4 shows the extinction efficiency $Q_{\text {ext }}$ for two silver nanoparticles of radius $a=50 \mathrm{~nm}$ with the interparticle spacing $d / a$ $=0.1$. In this case, there are three resonant peaks and one resonant dip. The corresponding real part of the $H_{y}$ component $\left(y\right.$ being the direction of $\mathbf{H}_{0}$ ) in the insets clearly show the dipole oscillation at $\lambda=516 \mathrm{~nm}$, quadrupole oscillation at $\lambda=370 \mathrm{~nm}$, and octopole oscillation at $\lambda=354 \mathrm{~nm}$, respectively. Note that higher order modes occur at short wavelengths with narrower bandwidths. This is consistent with the fact that the material absorption (or the imaginary part of the dielectric constant) for silver is smaller at shorter wavelengths (cf. Ref. [54]).

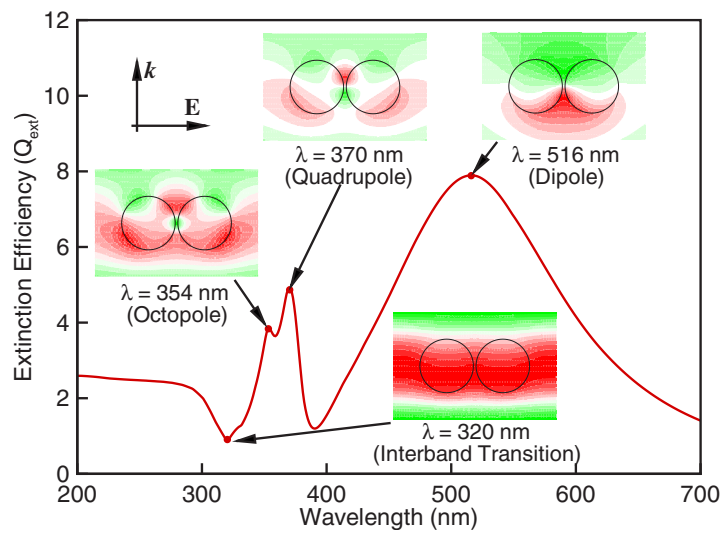

FIG. 4. (Color online) Extinction efficiency for two silver nanoparticles of radius $a=50 \mathrm{~nm}$ with interparticle spacing $d / a=0.1$. The insets show the real part of the $H_{y}$ component ( $y$ being the direction of $\mathbf{H}_{0}$ ) for the extinction peaks at $\lambda=516 \mathrm{~nm}$ (dipole), $370 \mathrm{~nm}$ (quadrupole), $354 \mathrm{~nm}$ (octopole), and for the extinction dip at $\lambda=320 \mathrm{~nm}$ (electronic interband transition). Red online color denotes positive field, and green online color denotes negative field.
Note also that in Fig. 4 there is an extinction dip located at $\lambda=320 \mathrm{~nm}$, where the electronic interband transitions occur. For silver, this happens at $318 \mathrm{~nm}$ or $3.9 \mathrm{eV}$ [3]. Due to the interband transitions, the electron-hole pairs are created in the nanoparticles by the absorption of photons, and they are bound together by the attractive Coulomb interactions [57]. As a result, the nanoparticles behave more like dielectrics rather than conductors, for the real part of the dielectric constant becomes positive at this wavelength [55]. In this situation, both the scattering and absorption efficiencies may attain their minimal values, resulting in an extinction dip. The real part of the $H_{y}$ component at $\lambda=320 \mathrm{~nm}$ is also shown in the inset of Fig. 4, where we observe that the nanoparticles appear to be somewhat transparent to the incident field. In fact, at this wavelength the dielectric constant $\varepsilon$ $=0.5175+0.688 i$ (experimental data from Ref. [54]) corresponds to a weakly adverse polarization $\left(-1<\chi_{e}<0, \chi_{e}\right.$ being the electric susceptibility), and the optical property of the nanoparticles lies between metals and pure dielectrics. For silver, the major particle plasmon resonances (including dipole, quadrupole, and octopole) occur at frequencies well below the electronic interband transitions. In this situation, silver nanoparticles can be treated as free-electron systems, and their optical properties are determined by the conduction electrons with only a constant real background polarizability associated with the core electrons [58].

Fundamental modes of particle plasmons can also be illustrated with surface charges on the nanoparticles. Figure 5 shows the surface charge distributions at two major resonances for the nanoparticle chain consisting of three silver particles of radius $a=25 \mathrm{~nm}$ with the interparticle spacing $d / a=0.1$, aligned parallel to the incident electric field. The wave vector $\mathbf{k}$ points into the page, and symmetric patterns of surface charges with respect to the particle chain axis are apparent. In Fig. 5(a), positive surface charges concentrate on one side of the particle and negative charges on the other side, exhibiting the dipole resonance pattern at $\lambda=456 \mathrm{~nm}$. Electric field vectors (with uniform vector length) at the center plane are overlaid on the figure to help illustrate the dipolar nature of plasmon resonances on particle chains. Note that in each particle the field vector directions tend towards the same direction. Further, the surface charges are most intense near the gaps between particles, and the distributions of positive and negative charges are somewhat different on the end particles due to the interparticle coupling with the center particle. However, each nanoparticle should remain electrically neutral, for the surface charges are all induced by polarizations.

Surface charge distribution associated with the second resonance at $\lambda=363 \mathrm{~nm}$ is shown in Fig. 5(b). For the two end particles, positive surface charges gather on two sides of the particle, separated by negative charges in between, or vise versa. This is a typical feature of quadrupole oscillation, and this behavior is also manifest on the electric fields in the particles. The field vectors approach horizontally (or vertically) from two sides, and orient vertically (or horizontally) towards the other two sides. For the center particle, the interparticle coupling comes from both neighboring particles, and the surface charge pattern shows locally a much higherorder resonance. The second resonance is therefore a com- 


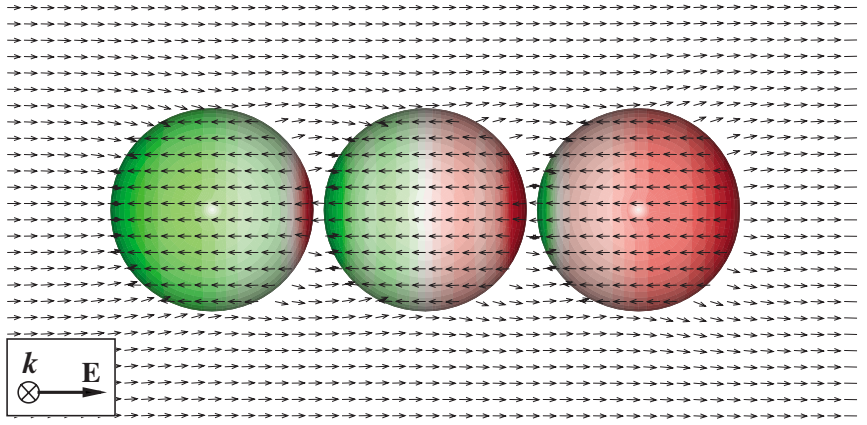

(a)

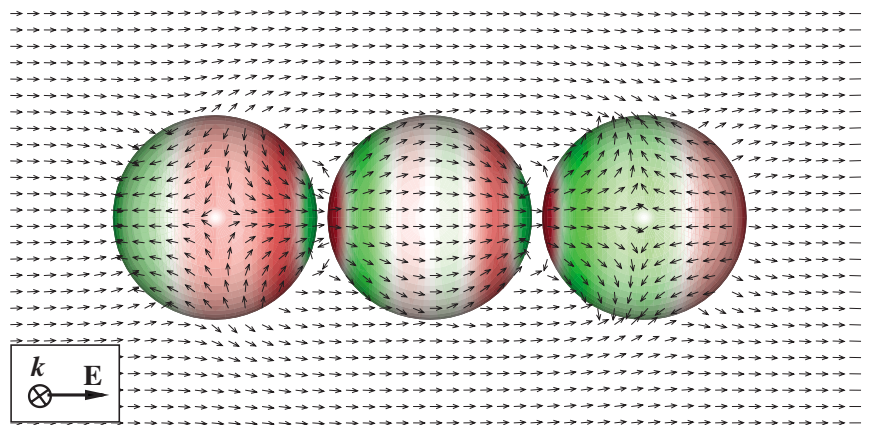

(b)

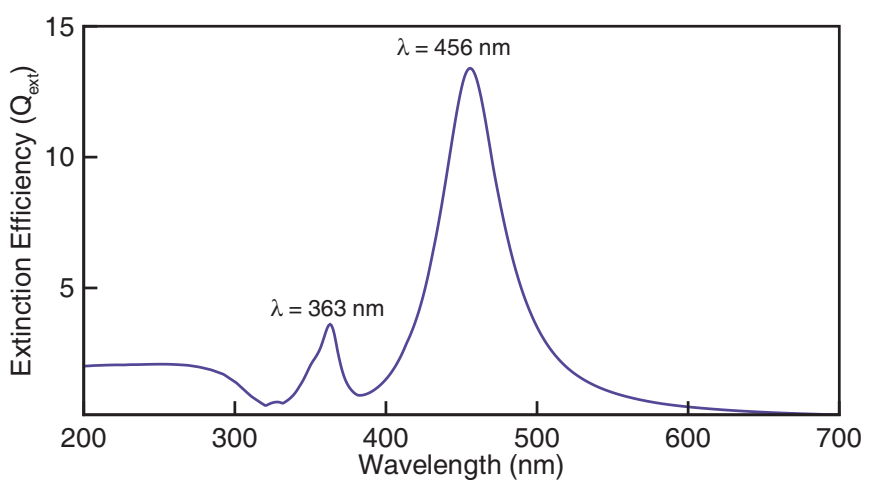

(c)

FIG. 5. (Color online) Surface charge distributions and electric field vectors for the nanoparticle chain consisting of three silver particles of radius $a=25 \mathrm{~nm}$ with interparticle spacing $d / a=0.1$. (a) Dipole resonance at $\lambda=456 \mathrm{~nm}$. (b) Composite higher-order resonance at $\lambda=363 \mathrm{~nm}$. Red online color denotes positive charge, and green online color denotes negative charge. The corresponding extinction spectrum is shown in (c).

posite higher-order oscillation. The corresponding extinction spectrum is shown in Fig. 5(c).

For more nanoparticles, interactions between particles allow a greater degree of freedom for plasmon resonances to occur. Figure 6 shows the surface charge distributions at two major resonances for six silver nanoparticles with the same size, spacing, and alignment as those in Fig. 5. Compared to the case of three particles, surface charge patterns for six particles become more complicated. Nevertheless, the dipole oscillation at $\lambda=504 \mathrm{~nm}$ can still be identified from the surface charge pattern in Fig. 6(a) for all particles. Due to the more complicated interparticle couplings, the second resonance at $\lambda=407 \mathrm{~nm}$ in Fig. 6(b) exhibits a composite oscil-

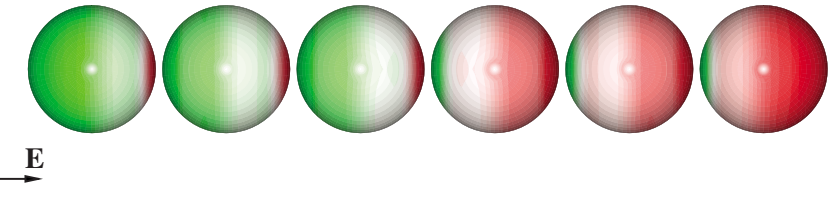

(a)

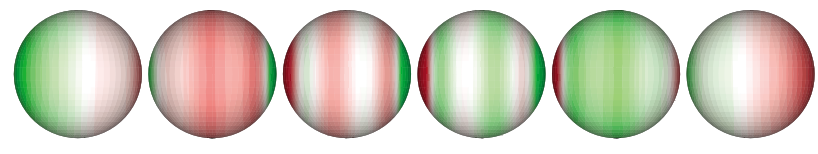
$\underset{\otimes}{\boldsymbol{k}} \mathbf{E}$

(b)

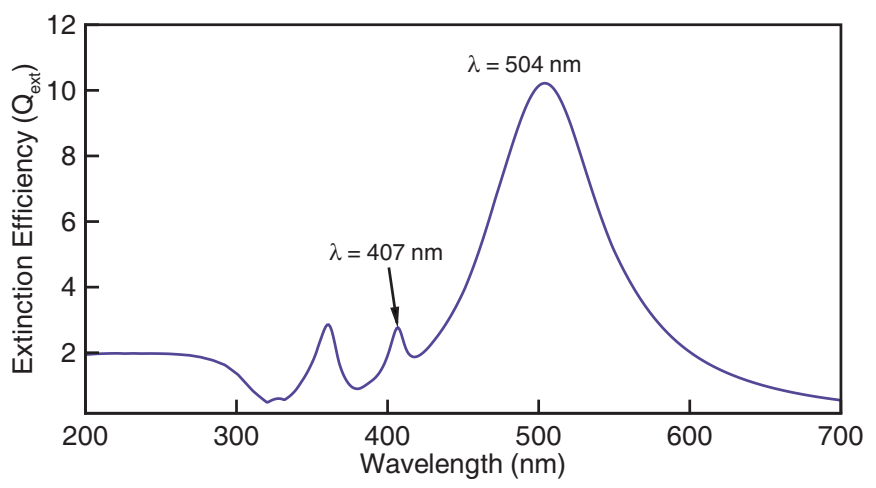

(c)

FIG. 6. (Color online) Surface charge distributions for the nanoparticle chain consisting of six silver particles of radius $a=25 \mathrm{~nm}$ with interparticle spacing $d / a=0.1$. (a) Dipole resonance at $\lambda$ $=504 \mathrm{~nm}$. (b) Composite higher-order resonance at $\lambda=407 \mathrm{~nm}$. Red online color denotes positive charge, and green online color denotes negative charge. The corresponding extinction spectrum is shown in (c).

lation in the nanoparticle chain. For the two end particles, a dipole oscillation appears, while for the two next inner particles, a quadrupole oscillation is present. As to the two center particles, a much higher-order mode similar to that in the three particles case is observed. The corresponding extinction spectrum is shown in Fig. 6(c).

The multipolar nature of plasmon resonances for nanoparticle chains can be further analyzed using the electric field enhancement $\left|\mathbf{E} / \mathbf{E}_{0}\right|$ along the chain axis, as Fig. 7 shows. The electric field is most intense on the particle surface as well as in the gap region, indicating a strong coupling between nanoparticles. For the first resonance at $\lambda=504 \mathrm{~nm}$, the electric field has the maximum enhancement $\left|\mathbf{E}_{\max } / \mathbf{E}_{0}\right|$ $=108$ at the center gap of the particle chain, and decreases toward the gaps on both ends. For the second resonance at $\lambda=407 \mathrm{~nm}$, the maximum field enhancement $\left|\mathbf{E}_{\max } / \mathbf{E}_{0}\right|=97$ occurs at the two end gaps, and a slightly smaller field enhancement appears at the center gap. It is found that the field enhancements across the gaps show complementary patterns for the two major resonances; the gaps with high enhancements for one resonance correspond to low enhancements for another, and vise versa. It is also noted that the maximum electric field always occurs at the nanoparticle surfaces (see the insets of Fig. 7) and decays much faster inside the nanoparticles than outside. 

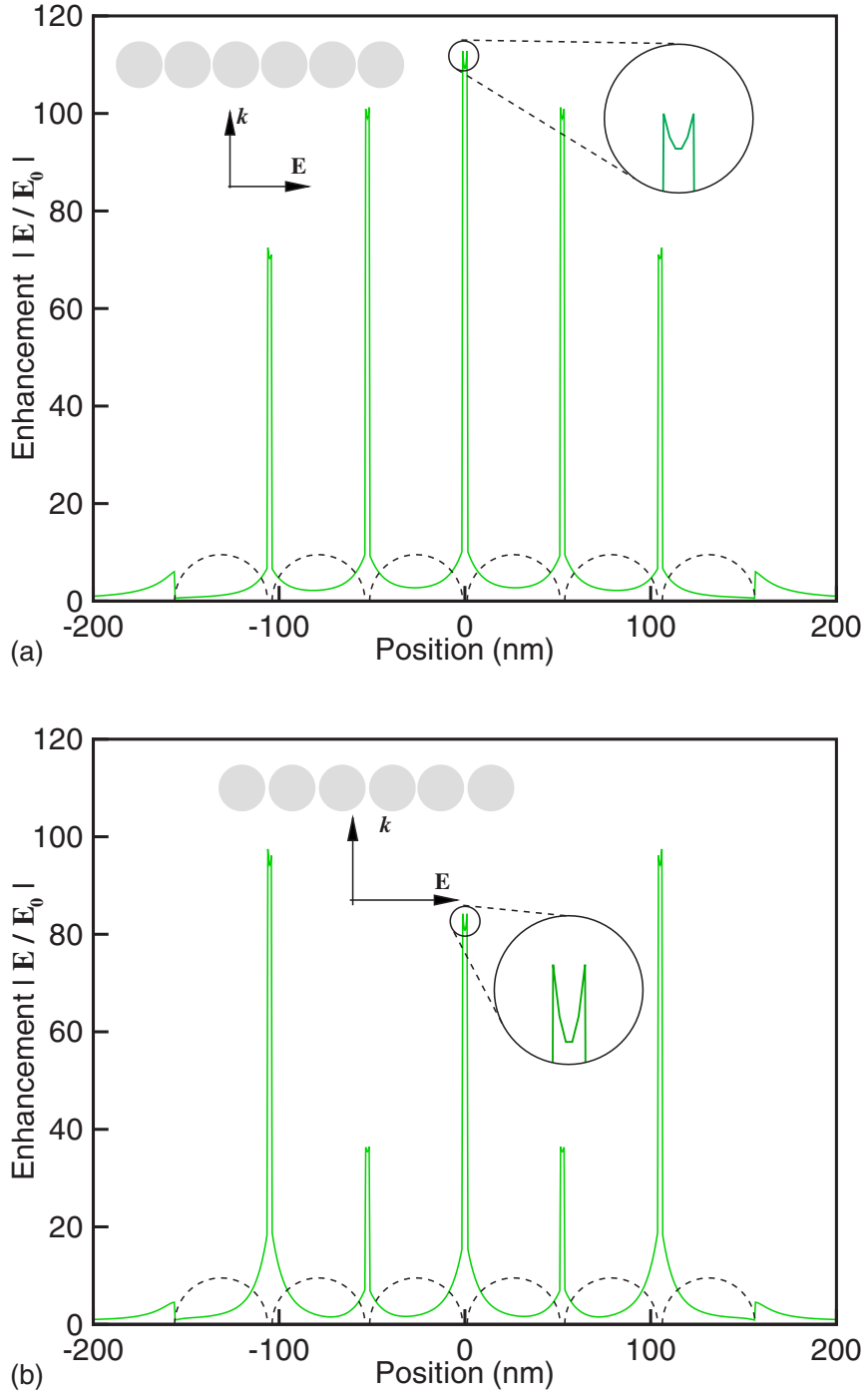

FIG. 7. (Color online) Distributions of the electric field enhancement $\left|\mathbf{E} / \mathbf{E}_{0}\right|$ along the chain axis for six silver nanoparticles of radius $a=25 \mathrm{~nm}$ with interparticle spacing $d / a=0.1$, aligned parallel to the incident electric field. (a) Dipole resonance at $\lambda$ $=504 \mathrm{~nm}$. (b) Composite higher-order resonance at $\lambda=407 \mathrm{~nm}$.

In another aspect, we present the case of nanoparticle chains consisting of different numbers of particles. Figure 8(a) shows the extinction efficiencies $Q_{\text {ext }}$ for the nanoparticle chains aligned parallel to the incident electric field (parallel alignment). It is shown that as the number of particles increases, the major extinction peaks move to larger wavelengths and become broadened. The frequency shift tends to saturate as the number of particles continues to increase. Additional minor extinction peaks associated with higher-order modes also occur at relatively shorter wavelengths. These additional modes are excited as parallel alignment could induce significant and complex charge interactions along the polarized particle chain. For a chain of three particles, there are two extinction peaks, while for 12 particles, four extinction peaks appear. Basically, the redshift, broadening, and additional resonances of particle plasmons for particle chains with increasing number of particles show qualitative trends similar to those for single particles with increasing particle
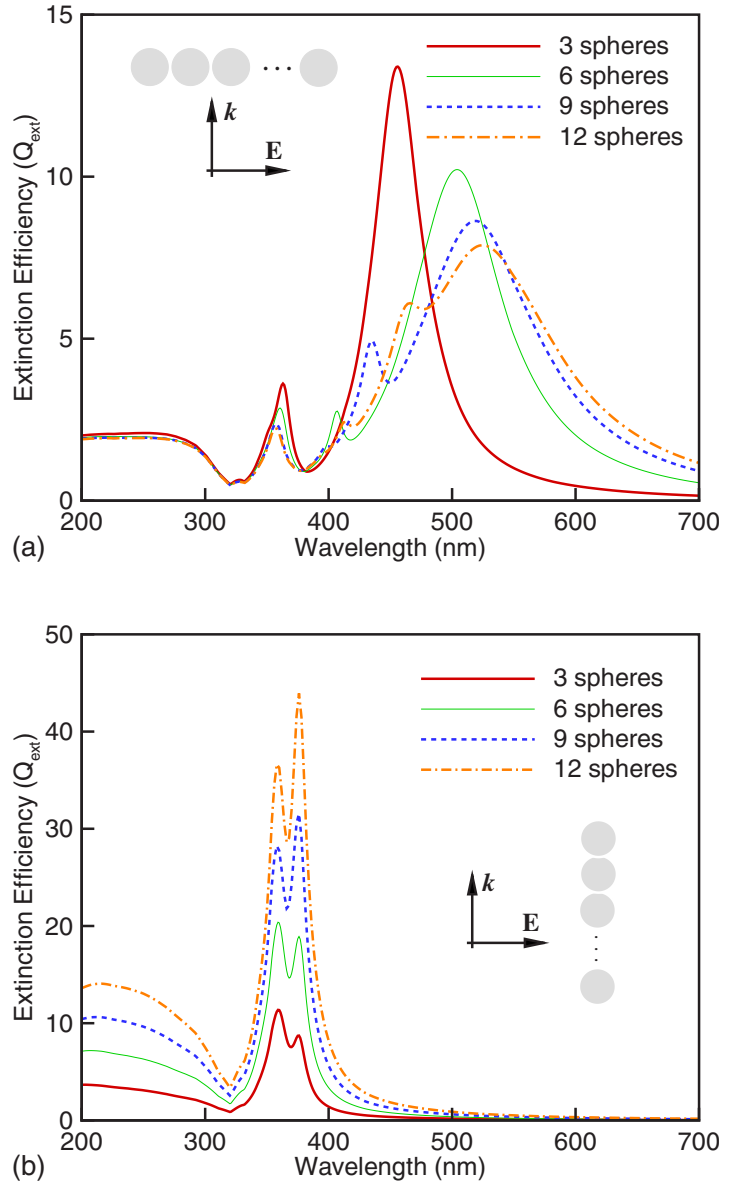

FIG. 8. (Color online) Extinction efficiencies for nanoparticle chains consisting of 3-12 silver particles of radius $a=25 \mathrm{~nm}$ with interparticle spacing $d / a=0.1$. (a) Particles aligned parallel to the incident electric field. (b) Particles aligned normal to the incident electric field.

size $[2,18,19]$. However, the underlying mechanism for nanoparticle chains is different due to the interparticle coupling between particles.

On the other hand, Fig. 8(b) shows very different behaviors for the same nanoparticle chains aligned normal to the incident electric field (normal alignment). Despite the number of particles, only two resonant peaks occur in this case. Note that the two peak frequencies are relatively closer to each other than in the case of parallel alignment. The two extinction peaks shift very little (dipole resonance at $\lambda$ $=372 \mathrm{~nm}$ and quadrupole resonance at $\lambda=358 \mathrm{~nm}$ ) as the number of particles changes. Since the particle size and the interparticle spacing are both much smaller than the wavelength, the retardation effect [31] is not significant. In this configuration, the particle chains appear to be the same size in the polarization direction of the electric field. The transversal polarization does not favor interparticle interactions, so the modes are associated more to individual particles, and therefore the addition of more particles does not change that much the nature of these transversal modes.

Finally, the effect of interparticle spacing for the nanoparticle chain is shown in Fig. 9 for six particles of radius $a$ $=25 \mathrm{~nm}$. For the parallel alignment as shown in Fig. 9(a), the 

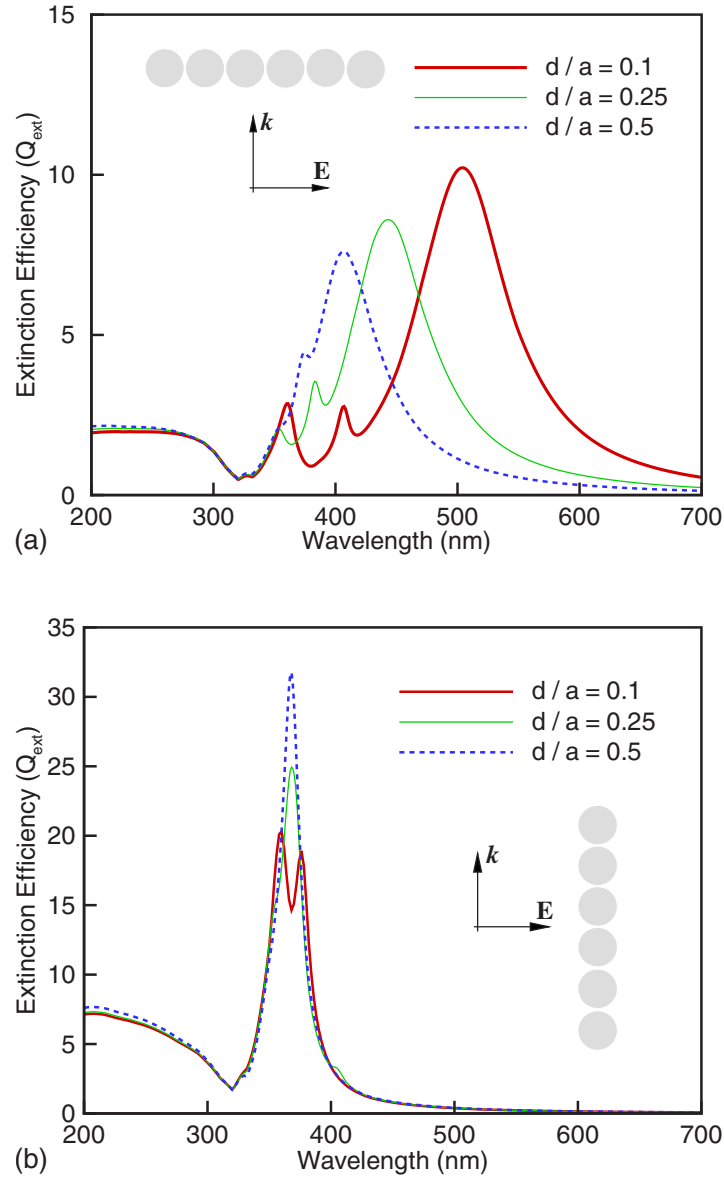

FIG. 9. (Color online) Extinction efficiencies for a nanoparticle chain consisting of six silver particles of radius $a=25 \mathrm{~nm}$ with various interparticle spacings. (a) Particles aligned parallel to the incident electric field. (b) Particles aligned normal to the incident electric field.

resonant peaks are redshifted, and additional higher-order modes with shorter wavelengths are excited as $d / a$ decreases. These features are similar to those for particle pairs with decreasing the interparticle spacing [32]. The interparticle coupling gives rise to distorted intraparticle charge distribution, and strong localized charges can occur near the gap. As a result, strong attractive interaction between these charges across the gap reduces the intraparticle restoring forces and lowers the resonant energies [29]. Notice that the peak broadening in this example is less significant, and the extinction patterns are roughly alike for different spacings. The frequency shift becomes even more significant as the spacing decreases further. For the normal alignment as shown in Fig. 9(b), however, the interparticle spacing has relatively little effect on the resonant peaks. For a larger $d / a$, there is only one resonant peak, but as $d / a$ decreases to 0.1 , two resonant peaks appear. The average frequency of the two resonant peaks $(365 \mathrm{~nm})$ is very close to that of the one resonance peak $(364 \mathrm{~nm})$.

\section{SUMMARY}

In conclusion, we have studied particle plasmons for a chain of silver nanoparticles by the multiple scattering method. The multiple scattering approach, presented in a fuller account, is shown to be an accurate and efficient formalism to solve for the electromagnetic fields and calculate the optical properties of multiple nanoparticles. In most of the examples discussed, the particles are aligned in parallel to the incident electric field (parallel alignment). For a chain of nanoparticles, we have presented surface charge patterns and electric field vectors to identify the multipolar nature of two major resonant modes. It is noted that the particle size (on the order of tens of nanometers) is relatively smaller than the wavelengths of the two major resonant modes (on the order of several hundred nanometers). The first extinction peak (with the lowest resonant frequency) is identified as a dipole resonance, while the second peak (with the next higher resonant frequency) is a composite higher-order resonance. It is shown that the electric field enhancement across the particle chain exhibits complementary patterns for the two major resonances. For the first resonance, the maximum field enhancement occurs at the center gap, while for the second resonance it appears at the two end gaps.

One focus of the present study is to examine the extinction spectrum to see how it is affected by the numbers of particles. First, we considered particles aligned parallel to the incident electric field (parallel alignment). It is shown that the extinction peaks move to longer wavelengths and become broadened as the number of particles increases. Meanwhile, additional higher-order modes are excited as parallel alignment could induce significant and complex charge interactions along the chain of polarized particles. Next, we considered particles aligned normally to the incident electric field (normal alignment). In this case, only two resonant peaks are apparent even though the number of particles increases. Moreover, the peak frequencies shift very little as the number of particles changes. For this alignment, the transversal polarization does not favor interparticle interactions, so the modes are associated more to individual particles, and therefore the addition of more particles does not change that much the nature of these transversal modes. The other primary focus of this study is to examine how the spectral shift is affected by the spacing of two neighboring nanoparticles in a chain. For the parallel alignment, the resonant peaks are redshifted, and higher-order resonances are excited as the interparticle spacing decreases. Peak broadening is less significant, and the extinction patterns are roughly alike for different spacings. For the normal alignment, only one resonance occurs for larger spacings, and the resonance splits into two resonances as the spacing decreases.

\section{ACKNOWLEDGMENTS}

The authors thank the anonymous reviewers for valuable information and suggestions in the revision of this paper. This work was supported in part by National Science Council of the Republic of China under Contracts No. NSC 942212-E-002-047 and No. NSC 95-2221-E-002-220, and the Ministry of Economic Affairs of the Republic of China under Contract No. MOEA 95-EC-17-A-08-S1-0006. 
[1] R. Ruppin, Phys. Rev. B 11, 2871 (1975).

[2] C. Bohren and D. Huffman, Absorption and Scattering by Small Particles (Wiley, New York, 1983).

[3] U. Kreibig and M. Vollmer, Optical Properties of Metal Clusters (Springer, New York, 1995).

[4] H. Raether, Surface Plasmons on Smooth and Rough Surfaces and on Grating (Springer-Verlag, Berlin, 1988).

[5] N. Peyghambarian, S. Koch, and A. Mysyrowicz, Introduction to Semiconductor Optics (Prentice-Hall, Englewood Cliffs, NJ, 1994).

[6] U. Kreibig, B. Schmitz, and H. D. Breuer, Phys. Rev. B 36, 5027 (1987).

[7] M. Ohtsu, K. Kobayashi, T. Kawazoe, S. Sangu, and T. Yatsui, IEEE J. Sel. Top. Quantum Electron. 8, 839 (2002).

[8] S. A. Maier, M. L. Brongersma, P. G. Kik, S. Meltzer, A. A. G. Requicha, and H. A. Atwater, Adv. Mater. (Weinheim, Ger.) 13, 2 (2001).

[9] S. Nie and S. R. Emory, Science 275, 1102 (1997).

[10] K. Kneipp, Y. Wang, H. Kneipp, L. T. Perelman, I. Itzkan, R. R. Dasari, and M. S. Feld, Phys. Rev. Lett. 78, 1667 (1997).

[11] C. Talley, J. Jackson, C. Oubre, N. Grady, C. Hollars, S. Lane, T. Huser, P. Nordlander, and N. Halas, Nano Lett. 5, 1569 (2005).

[12] D. A. Schultz, Curr. Opin. Biotechnol. 14, 13 (2003).

[13] T. R. Jensen, M. D. Malinsky, C. L. Haynes, and R. P. V. Duyne, J. Phys. Chem. B 104, 10549 (2000).

[14] M. D. Malinsky, K. L. Kelly, G. C. Schatz, and R. P. V. Duyne, J. Phys. Chem. B 105, 2343 (2001).

[15] J. Tominaga, C. Mihalcea, D. Buechel, H. Fukuda, T. Nakano, and N. Atoda, Appl. Phys. Lett. 78, 2417 (2001).

[16] W. C. Liu, C. Y. Wen, K. H. Chen, W. C. Lin, and D. P. Tsai, Appl. Phys. Lett. 78, 685 (2001).

[17] F. Fujimoto and K. Komaki, J. Phys. Soc. Jpn. 25, 1679 (1968).

[18] J. P. Kottmann and O. J. F. Martin, Opt. Express 6, 213 (2000).

[19] J. P. Kottmann, O. J. F. Martin, D. R. Smith, and S. Schultz, Phys. Rev. B 64, 235402 (2001).

[20] R. Ruppin, Phys. Rev. B 26, 3440 (1982).

[21] G. B. Smith, W. E. Vargas, G. A. Niklasson, J. A. Sotelo, A. V. Paley, and A. V. Radchik, Opt. Commun. 115, 8 (1995).

[22] M. Schmeits and L. Dambly, Phys. Rev. B 44, 12706 (1991).

[23] N. Zabala, A. Rivacoba, and P. M. Echenique, Phys. Rev. B 56, 7623 (1997).

[24] R. Rojas and F. Claro, Phys. Rev. B 34, 3730 (1986).

[25] R. Fuchs and F. Claro, Phys. Rev. B 39, 3875 (1989).

[26] J. Aizpurua, A. Rivacoba, N. Zabala, and F. J. Garcia de Abajo, Surf. Sci. 402, 418 (1997).

[27] H. Tamaru, H. Kuwata, H. Miyazaki, and K. Miyano, Appl. Phys. Lett. 80, 1826 (2002).

[28] H. Xu, J. Aizpurua, M. Kall, and P. Apell, Phys. Rev. E 62, 4318 (2000).
[29] J. Aizpurua, G. W. Bryant, L. J. Richter, F. J. Garcia de Abajo, B. K. Kelley, and T. Mallouk, Phys. Rev. B 71, 235420 (2005).

[30] H. Xu and M. Kall, Phys. Rev. Lett. 89, 246802 (2002).

[31] J. P. Kottmann and O. J. F. Martin, Opt. Lett. 26, 1096 (2001).

[32] J. P. Kottmann and O. J. F. Martin, Opt. Express 8, 655 (2001).

[33] K. H. Su, Q. H. Wei, X. Zhang, J. J. Mock, D. R. Smith, and S. Schultz, Nano Lett. 3, 1087 (2003).

[34] W. Rechberger, A. Hohenau, A. Leitner, J. R. Krenn, B. Lamprecht, and F. Aussenegg, Opt. Commun. 220, 137 (2003).

[35] L. Zhao, K. Kelly, and G. Schatz, J. Phys. Chem. B 107, 7343 (2003).

[36] I. Romero, J. Aizpurua, G. Bryant, and F. J. Garcia de Abajo, Opt. Express 14, 9988 (2006).

[37] K. Kelly, E. Coronado, L. Zhao, and G. Schatz, J. Phys. Chem. B 107, 668 (2003).

[38] M. Quinten, A. Leitner, J. R. Krenn, and F. R. Aussenegg, Opt. Lett. 23, 1331 (1998).

[39] D. Citrin, Nano Lett. 4, 1561 (2004).

[40] D. Citrin, Nano Lett. 5, 985 (2005).

[41] D. Citrin, Opt. Lett. 31, 98 (2006).

[42] S. A. Maier, P. G. Kik, and H. A. Atwater, Phys. Rev. B 67, 205402 (2003).

[43] L. A. Sweatlock, S. A. Maier, H. A. Atwater, J. J. Penninkhof, and A. Polman, Phys. Rev. B 71, 235408 (2005).

[44] S. Y. Park and D. Stroud, Phys. Rev. B 69, 125418 (2004).

[45] E. Prodan, C. Radloff, N. Halas, and P. Nordlander, Science 302, 419 (2003).

[46] P. Nordlander, C. Oubre, E. Prodan, K. Li, and M. I. Stockman, Nano Lett. 4, 899 (2004).

[47] H. Wang, Y. Wu, B. Lassiter, C. Nehl, J. Hafner, P. Nordlander, and N. Halas, Proc. Natl. Acad. Sci. U.S.A. 103, 10856 (2006).

[48] H. Wang, D. Brandl, P. Nordlander, and N. Halas, Acc. Chem. Res. 40, 53 (2007)

[49] D. W. Mackowski, Proc. R. Soc. London, Ser. A 433, 599 (1991).

[50] K. A. Fuller, Appl. Opt. 30, 4716 (1991).

[51] S. Kawata, Near-Field Optics and Surface Plasmon Polaritons (Springer, New York, 2001).

[52] P. Apell and D. R. Penn, Phys. Rev. Lett. 50, 1316 (1983).

[53] D. W. Mackowski, J. Opt. Soc. Am. A 11, 2851 (1994).

[54] P. B. Johnson and R. W. Christy, Phys. Rev. B 6, 4370 (1972).

[55] A. Pinchuk, G. Plessen, and U. Kreibig, J. Phys. D 37, 3133 (2004).

[56] S. L. Westcott, J. B. Jackson, C. Radloff, and N. J. Halas, Phys. Rev. B 66, 155431 (2002).

[57] C. Kittel, Introduction to Solid State Physics, 7th ed. (Wiley, New York, 1996).

[58] H. Wang, F. Tam, N. Grady, and N. Halas, J. Phys. Chem. B 109, 18218 (2005). 